\title{
Embedded Image Compression: A Review
}

\author{
Ruchita K. Ingole \\ Department of Electronics \& Telecommunication Engineering, G. H. Raisoni College of Engineering \& Management, Amravati, \\ Maharashtra, India
}

\section{Email address:}

ruchitaingole123@gmail.com (R. K. Ingole)

\section{To cite this article:}

Ruchita K. Ingole. Embedded Image Compression: A Review. International Journal of Data Science and Analysis. Vol. 3, No. 1, 2017, pp. 1-4. doi: 10.11648/j.ijdsa.20170301.11

Received: October 19, 2016; Accepted: November 24, 2016; Published: March 21, 2017

\begin{abstract}
In this paper, the various research papers related to 'embedded image compression' are studied. The aim of the present work is to study the methods adopted and analysis and results obtained. The different methods are adopted by various researchers. The present paper includes discrete wavelet transform (DWT), such as embedded zero wavelet (EZW) and the set partitioning in hierarchical trees (SPIHT) are studied. Block based discrete cosine transform (DCT) encoders are used in many image and video coding standards. Wavelet-based image coders such as embedded zero tree wavelet (EZW) coder, set partitioning in hierarchical trees (SPIHTs), set partitioning embedded block (SPECK), morphological representations of wavelet data (MRWD) and significance-linked connected component analysis (SLCC) are also the part of embedded image compression. Different types of redundancy present in an image, such as Spatial Redundancy, Statistical Redundancy and Human Vision Redundancy are very necessary for analysis. The JPE -2OOU image compression standard is increasingly gaining widespread importance. Ultra spectral imaging is a relatively recent development which makes quantitative remote sensing of the Earth's surface possible.
\end{abstract}

Keywords: Embedded Image, Wavelet Transform, Image Coder, Compression Standard and Redundancy

\section{Introduction}

Ultra spectral imaging is a relatively recent development which makes quantitative remote sensing of the Earth's surface possible. Images are acquired by sensors and their characteristics are determined by sensor capabilities. Each image is a high resolution three dimensional cube containing both spatial and spectral information about the zone under acquisition [1].

The JPE -2OOU image compression standard is increasingly gaining widespread importance. The rich variety of features makes it highly suitable for a large spectrum of applications but at the same time its associated complexion makes it hard 60 optima e for particular parts. One the key step during the processing is entropy coding that takes about 0 of the execution time [2].

Compression is necessary in medical image preservation because of the huge data quantity. Medical images are different from the common images because of their own characteristics, for example, part of information in CT image is useless, and it's a kind of resource waste to save this part information [3].
Block based discrete cosine transform (DCT) encoders are used in many image and video coding standards as a result of their high decorrelation performance and the availability of fast DCT algorithms enabling real-time implementation. While DCT block coding provides good reproduction without noticeable artifacts at high or moderate bit rates, at low bit rates reconstructed images usually suffer from visual artifacts [4].

In the wide range applications of wireless sensor network (WSN), sensor nodes are located in certain environments where it is hard to access and limited power supplied by small irreplaceable batteries. Sensor node devices can only transmit a finite number of data packets in their lifetime. More recently, increasing interest and more attention has risen for ubiquitous applications requiring vision capabilities [6].

There is a real need of energy-autonomous sensory systems organized in wireless sensor networks, aimed at monitoring many different physical quantities in our everyday life environment. Although, big advantages have been done in all those applications requiring relatively simple sensors (temperature, pressure, humidity, light, sound, etc.), no significant steps forward have been made toward more 
complex sensors, like vision sensors, where the information is much more dense and the typical activity is larger, as much as their output bandwidth [7].

Data compression is the art or since of representing information in a compact form i.e. reduces the size of the data to reduce storage space and transmission bandwidth. Image compression reducing the amount of data required to represent a digital image by removing redundant or non-vital data. There are different types of redundancy present in an image, such as Spatial Redundancy, Statistical Redundancy and Human Vision Redundancy [8].

Nowadays, High speed image-sensors with high resolution are more and more required in new applications, such as broadcasting, observation of high-speed phenomena, analysis of high-speed machinery and so on, making on-sensor analog todigital conversion necessary. However, different types of ADC reveal a trade-off between speed, resolution and power. Flash $\mathrm{ADC}$ is the fastest one with a limited 6 8-bit resolution [9].

Proposed embedded eye tracking system is a bright example of human-machine interaction system, which allows user to interact with objects, which are placed in dedicated environment, only by looking on them. The system consists of wearable Weetsy glasses (equipped with miniaturized camera sensor), Weetsy board (intermediate component for preliminary processing; implemented as an embedded system) and processing unit (implemented in PC), which is used for most of the processing needed to find coordinates of user's pupil [10].

Hand held mobile or portable devices have limited memory, processing power and battery life. Real-time processing and transmission of images using these devices require an image coding algorithm that can compress efficiently with reduced complexity. Wavelet-based image coders such as embedded zero tree wavelet (EZW) coder, set partitioning in hierarchical trees (SPIHTs), set partitioning embedded block (SPECK), morphological representations of wavelet data (MRWD) and significance-linked connected component analysis (SLCCA) provide excellent rate distortion (RD) performances by exploiting magnitude correlation within or across bands of decomposition [11].

The embedded image coders provide SNR scalability, i.e., they can provide fine bit rate control and progressive transmission. Most of embedded image coders consist of three parts: significance coding with successive approximation quantization, sorting algorithm, and arithmetic coding. In this kind of coders, the significance of each coefficient is encoded, and the coefficients identified as significant in the previous biplanes are progressively refined [12].

Recently, a lot of work has been dedicated to efficient transmission of images in multimedia communications over wireless channels. Image compression algorithms based on discrete wavelet transform (DWT), such as embedded zero wavelet $(\mathrm{EZW})$ and the set partitioning in hierarchical trees (SPIHT) provide excellent rate distortion performance with low encoding complexity. However, it is quite fragile against bit errors in wireless channels because a single bit error propagation and possible loss of synchronization [13].
Driven by a growing demand for transmission and storage of visual data over media with limited capacity, increasing efforts have been devoted to improve compression techniques for visual information. The Discrete Wavelet Transform (DWT) has matured and has become very effective for the compression of natural images. Since the introduction of the zero tree wavelet images coding by Shapiro, several coding algorithms have been developed. Said and Pearlman proposed an improved scheme, called Set Partitioning in Hierarchical Trees (SPIHT) [14].

\section{Compression Techniques}

\subsection{Image Compression}

To reduce time of image transmission from Weetsy board to processing unit over a network, image compression could be employed. As a result, images will be sent in compact (compressed) form. Therefore, less bytes will be used for transmission.

\section{Fractal Image Compression}

Fractal is one effective method to describe natural modality in the process of transformation and iteration. In 1973, Benoit Mandelbrot firstly brought forward the idea of fractal geometry, Infinity self similarity is the soul of fractal [18].

\subsection{EZW Algorithm}

The Ezw encoder was originally designed to operate on images (2D signals) but it can also be used on other dimensional signals. It is based on progressive encoding to compress an image into a bit stream with increasing accuracy. This means that when more bits are added to the stream, the decoded image will contain more detail, a property similar to JPEG encoded images.

\subsubsection{Decomposition of Sub Bands}

After generating wavelet coefficients and converting it to matrix form the encoding must be done. Before encoding hierarchical sub band decomposition has to be done [16].

\subsubsection{Scanning Order}

In order to encode the coefficients scanning has to be done and the lower sub bands should be scanned completely before going to the higher sub bands. The scan order seems to be of some influence of the final compression results.

\subsection{SPIHT Algorithm}

SPIHT is having embedded coding property it sorts the information on demand and requirement for powerful error correction codes decreases from the beginning to the end of the compressed file. If an error is detected but not corrected then decoder rejected that data after point and still displays the image obtained with the bits received before the error.

\subsection{Huffman Encoding}

Huffman coding is an entropy encoding algorithm used for 
lossless data compression. The term refers to the use of a variable -length code table for encoding a source symbol (such as a character in a life where the variable-length code table has been derived in a particular way based on the estimated probability of occurrence for each possible value of the source symbol.

\subsection{Lossless Compression}

Lossless compression technique involves no loss of information. If data have been lossless compressed the original data can be recovered exactly from the compressed data, Lossless compression is generally used for applications that cannot tolerate any difference between the original and reconstructed data [8].

\subsection{Near Lossless Compression}

Near lossless compression technique is a lossy compression method where the reconstructed pixels differ from the original pixels by no more than a predetermined value, in near-lossless compression data is guaranteed to be within a specified range based on the near-lossless threshold [8].

\subsection{Lossy Compression}

Lossy compression reduces the original image size by removing non-vital information, i.e., this technique involves some loss of information and data that have been compressed, using lossy techniques generally cannot be recovered or reconstructed exactly [8].

\section{Different Methods}

\subsection{Discrete Tchebichef Transform (DTT)}

The DTT is relatively a new transform that uses the Tchebichef moments to provide a basis matrix. As with DCT, the DTT is derived from the orthonormal Tchebichef polynomials [11]. For image of size $\mathrm{N} \times \mathrm{N}$, the forward DTT of order $\mathrm{u}+\mathrm{v}$ is defined as

$$
\mathrm{T}_{\mathrm{uv}}=\sum_{\mathrm{x}=0}^{\mathrm{N}-1} \sum_{\mathrm{y}=0}^{\mathrm{N}-1} \mathrm{t}_{\mathrm{u}}(\mathrm{x}) \mathrm{t}_{\mathrm{v}}(\mathrm{y}) \mathrm{f}(\mathrm{x}, \mathrm{y})
$$

Where $u, v=0,1,2 \ldots N-1$. The inverse transform of DTT is defined by

$$
f(x, y)=\sum_{u=0}^{N-1} \sum_{v=0}^{N-1} T_{u v} t_{u}(x) t_{v}(y)
$$

Where $\mathrm{x}, \mathrm{y}=0,1,2 \ldots \mathrm{N}-1$

\subsection{Discrete Wavelet Transforms (DWT) Implementation}

There are two main methods for DWT implementation: Convolution Filter Banks which is the earlier method of DWT implementation and the Lifting Scheme which is newer method for constructing and performing wavelets.

\subsection{Convolution Filter Bank Based DWT}

This is an earlier implementation of the traditional DWT, by means of the Mallat filter bank scheme which includes two main steps: signal filtering and decimating with a couple of Quadrature Mirror Filters (QMFs). The filter-bank can be realized using FIR filters.

\subsection{Lifting Scheme (Ls) Based DWT}

The convolution-based 1-D DWT requires a large number of arithmetic computations and a large memory for storage. Such features are not desirable for either high speed or lowpower image processing applications in severely constrained hardware environments such as WSNs [6].

\subsection{Set-Partitioning in Hierarchical Trees (SPIHT) Image Compression Implementation}

SPIHT is an advanced version of EZW algorithm, where coefficient-trees are processed in a more efficient way. In SPIHT image compression, an image is first wavelet transformed before the set-partitioning coding is applied.

\section{Performance Parameters of Image Compression}

\subsection{Error Metrics}

Mean square error (MSE) is a criterion for an estimator the choice is the one that minimizes the sum of squared errors due to bias and due to variance.

\subsection{Compression Metrics}

Compression ratio $(\mathrm{CR})$ is defined as the ratio between the uncompressed image size and compressed image size [15].

\section{Conclusion}

The present work includes the study of discrete wavelet transform (DWT), such as embedded zero wavelet (EZW) and the set partitioning in hierarchical trees (SPIHT). This study is very essential to do the further research in the embedded image compression.

\section{References}

[1] Julia Minguillon, Jordi Herrera-Joancomart, Joan SerraSagrista and Fernando Garcia-Vilchez, "Influence of Mark Embedding Strategies on Lossless Compression of Ultraspectral Images", IEEE, vol. 4, issue 5, 2005.

[2] I. Aouadi and O. Hammami, "Low Power JPEG-2000 Image Compression for Industrial Embedded Applications", IEEE International Conference on industrial Technology (ICIT), 2004.

[3] Li Guoli, Zhang Jian, Wang Qunjing, Hu Cungang, Deng Na And Li Jianping, "Application of Region Selective Embedded Zerotree Wavelet Coder in CT Image Compression", Proceedings of the 2005 IEEE Engineering in Medicine and Biology 27th Annual Conference Shanghai, China, September $1-4,2005$. 
[4] Oguzhan Urhan and Sarp Ertürk, "Parameter Embedding Mode and Optimal Post-Process Filtering for Improved WDCT Image Compression", IEEE Transactions On Circuits And Systems For Video Technology, Vol. 18, No. 4, April 2008.

[5] Shaowei Li and Xinqing Zhuang, "Embedded Based Radar Image Acquisition and Compression", International Conference on Electronic \& Mechanical Engineering and Information Technology, 2011.

[6] Khamees Khalaf Hasan and Umi Kalthum Ngah, "The Most Proper Wavelet Filters in Low-Complexity and an Embedded Hierarchical Image Compression Structures for Wireless Sensor Network Implementation Requirements", IEEE International Conference on Control System, Computing and Engineering, Penang, Malaysia, 23 - 25 Nov. 2012.

[7] Nicola Cottini, Leonardo Gasparini, Marco De Nicola, Nicola Massari and Massimo Gottardi, "A CMOS Ultra-Low Power Vision Sensor With Image Compression and Embedded Event-Driven Energy-Management”, IEEE Journal On Emerging And Selected Topics In Circuits And Systems, Vol. 1, No. 3, September 2011.

[8] Ali A. Al-hamid, Ahmed Yahya and Reda A. El-Khoribi, "Optimized Image Compression Techniques for the Embedded Processors", International Journal of Hybrid Information Technology Vol. 9, No. 1, pp. 319-328, 2016.

[9] Mengyun Yue, Dong Wu and Zheyao Wang, "A 15-bit TwoStep Sigma-Delta ADC with Embedded Compression for Image Sensor Array", IEEE, vol. 3, issue 13, 2013.

[10] Pavel Morozkin, Marc Swynghedauw and Maria Trocan, “An Image Compression for Embedded Eye-Tracking Applications", vol. 4, issue 16, Journal of European Union, 2016.

[11] Ranjan Kumar Senapati, Umesh C. Pati and Kamala Kanta Mahapatra, "Reduced memory, low complexity embedded image compression algorithm using hierarchical listless discrete Tchebichef transform", journal of IET Image Process., Vol. 8, Iss. 4, pp. 213-238, 2014.

[12] Han Sae Song and Nam Ik Cho, "DCT-Based Embedded Image Compression With a New Coefficient Sorting Method", IEEE Signal Processing Letters, Vol. 16, No. 5, May 2009.

[13] Srikanth and Sukadev Meher, "Compression Efficiency for Combining Different Embedded Image Compression Techniques with Huffman Encoding", International conference on Communication and Signal Processing, April 35, India, 2013.

[14] Li Zhu and Yi min Yang, "Embeded Image Compression Using Differential Coding and Optimization Method", IEEE, issue $11,2011$.

[15] Ali A. Al-hamid, Ahmed Yahya and Reda A. El-Khoribi, "Optimized Image Compression Techniques for the Embedded Processors", International Journal of Hybrid Information Technology, Vol. 9, No. 1, pp. 319-328, 2016.

[16] Yasaswi Velamuri, Sandhya Patnayakuni and Nancharaiah Vejendla, "Compression Efficiency of Different Embedded Image Compression Techniques with Huffman Encoding", International Journal of Engineering Research and Development, Volume 9, Issue 12, PP. 13-20, February 2014.

[17] Rehna V. J, and Jeya Kumar M. K, "Wavelet Based Image Coding Schemes: A Recent Survey", International Journal on Soft Computing (IJSC) Vol. 3, No. 3, August 2012.

[18] Pardeep Singh, Sugandha Sharma and Bhupinder Singh, "Comparative analysis of Embedded Zero tree and Fractal Image Compression Techniques", International Journal of Advanced Research in Computer Science and Software Engineering, Volume 2, Issue 2, February 2012. 